\title{
Mão na Massa: Dinâmica Vivencial para Apoio ao Ensino de Gerenciamento de Projetos de Software
}

\author{
Pablo Schoeffel ${ }^{1}$, Raul Sidnei Wazlawick ${ }^{2}$ \\ ${ }^{1}$ Departamento de Engenharia de Software \\ Universidade do Estado de Santa Catarina (UDESC) - Ibirama, SC - Brasil \\ ${ }^{2}$ Departamento de Informática e Estatística \\ Universidade Federal de Santa Catarina (UFSC) - Florianópolis, SC - Brasil \\ pabloschoeffel@yahoo.com, wazlawick@gmail.com
}

\begin{abstract}
As Project Management teaching is usually theoretical, approaches to motivate students and help them to practice those concepts are considered necessary. This paper describes experimental activities to support project management teaching, which consist of a team developing a complete software project, from its beginning to its end including management practices. The paper describes the activities in detail, as well as a proof of concept including a posttest evaluation made with 35 graduation students from two Software Engineering classes. The study produced positive results in terms of motivation and learning, implying that this approach is really effective.
\end{abstract}

Resumo. Como o ensino de Gerenciamento de Projetos é essencialmente teórico, requer de abordagens que permitam aos alunos praticarem os conceitos e se motivarem para a disciplina. Este artigo descreve uma dinâmica vivencial para apoiar o ensino de gerenciamento de projetos, que consiste no desenvolvimento de um projeto de software completo, em equipe, do início ao fim, vivenciando as práticas de gerenciamento. $O$ trabalho descreve o detalhamento da dinâmica, assim como o relato de aplicação $e$ uma avaliação pós-teste realizada com 35 alunos de duas turmas de um curso de Engenharia de Software. O estudo identificou resultados positivos tanto em aspectos motivacionais quanto de aprendizagem, sugerindo a efetividade da atividade.

\section{Introdução}

As estatísticas mostram que os projetos de Tecnologia da Informação continuam tendo um índice significativo de falhas e cancelamentos. Somente $39 \%$ dos projetos foram concluídos com sucesso e 18\% cancelados (STANDISH GROUP, 2013).

Embora, segundo Tatnall e Reyes (2005), a maioria dos guias curriculares modelos dar um peso significativo para o ensino de Gerenciamento de Projetos (GP), pode-se supor que o ensino do tema não está ou está afetando num grau pequeno a utilização de melhores práticas nos projetos reais, aumentando o sucesso destes.

Segundo Ibrahim (2011), a disciplina de GP é teórica, em sua natureza, sendo um desafio ensinar seu conteúdo, uma vez que pode ser desinteressante quando ensinado teoricamente. Ojiako et al. (2011) diz que iniciativas de ensino e aprendizagem em GP 
requerem novas e não tradicionais formas de pensar. "Um dos principais desafios no ensino de Engenharia de Software é proporcionar aos alunos experiências significativas, os quais irão achar úteis quando entram no mercado de trabalho" (BOVOTA, 2012).

Atividades práticas permitem que o aluno seja mais proativo e se envolva ao mesmo tempo em que aprende. A combinação de várias abordagens pode ser considerada uma chave para facilitar o processo de ensino e aprendizagem (Ibrahim, 2011). Segundo NTL Institute for Applied Behavioral Science (?), a atividade prática tem um desempenho melhor (75\%) que a maioria das demais, ficando abaixo somente de ensinar outras pessoas.

Uma dessas abordagens práticas, criada por Kolb (1984), é definida como a Teoria da Aprendizagem Experiencial ou Vivencial e é o processo pelo qual o conhecimento é criado através da transformação da experiência. O conhecimento resulta da combinação de compreender e transformar a experiência" (Kolb, 1984).

Percebe-se os grandes desafios no ensino de GP e na necessidade de utilizar diferentes abordagens, com ênfase para abordagens práticas. Foram encontrados diversos trabalhos que utilizam diferentes abordagens para o ensino de GP, como: jogos (Junior e Petri, 2014), simuladores (Dantas, Barros e Werner, 2004) e atividades vivenciais (Cidral, 2003; Schoeffel, 2014). As principais contribuições desse artigo frente aos trabalhos similares é a vivência de um projeto completo de software, passando por todas as etapas, e aplicado em um curto espaço de tempo numa disciplina de pós-graduação.

Esse trabalho visa demonstrar a dinâmica vivencial Mão na Massa, criada para explorar o ensino do gerenciamento de projetos por meio da prática, assim como um relato de sua aplicação em duas turmas de um curso de Pós-Graduação lato sensu em Engenharia de Software. O artigo está organizado da seguinte forma: na Seção 2 são apresentados os principais trabalhos correlatos, na Seção 3 é apresentada a metodologia utilizada, na Seção 4 é descrita a dinâmica vivencial, na Seção 5 são mostrados os resultados da aplicação da atividade e na Seção 6 são descritas as considerações finais.

\section{Trabalhos Correlatos}

O trabalho descrito em Corrêa et al. (2011) relata uma abordagem vivencial como ferramenta aplicada ao ensino de Gerenciamento de Projetos, cujo escopo do projeto é uma festa de formatura. A abordagem proposta abrange somente o planejamento e é desenvolvida ao longo de um semestre, com alunos de graduação.

Cano et al. (2006) descreve a aplicação de uma abordagem para treinamento em Gerenciamento de Projetos baseada na resolução de problemas de clientes reais. $\mathrm{O}$ trabalho não deixa claro o escopo dos projetos, mas sim a metodologia utilizada. Também é focada para alunos de graduação e desenvolvida durante todo o semestre.

Cook e Olson (2006) relata uma dinâmica intitulada "The Sky's the Limit", cujo objetivo é ensinar GP por meio do planejamento e construção de uma estrutura com materiais fornecidos. É uma atividade rápida (cerca de 60 minutos) e os objetivos de aprendizagem são conceitos básicos de acompanhamento e planejamento de projetos.

Outro trabalho é o de Cidral (2003), que descreve uma "metodologia de aprendizagem vivencial para o desenvolvimento de competências para o gerenciamento da implementação de sistemas de informação". Trata da forma de aplicação de uma 
abordagem vivencial para TI, aplicada em 124 alunos de graduação. A atividade é baseada em simulações de problemas reais de projetos de implantação de software e os resultados das avaliações com os participantes foram positivos.

Schoeffel (2014) descreve a dinâmica vivencial PizzaMia, que tem por objetivo simular a execução de um projeto, baseado no PMBOK, na realização de uma refeição pelos alunos. O trabalho mostra os resultados na aplicação com 35 alunos de duas turmas de um curso de especialização, mostrando resultados positivos tanto na motivação quanto na aprendizagem.

Esse trabalho descreve uma dinâmica vivencial para apoio ao ensino de gerenciamento de projetos baseado na execução de um projeto de software, do início ao fim. Como diferenciais dos trabalhos correlatos encontrados, a dinâmica proposta não é uma simulação, mas uma execução completa de um projeto; permite sua execução em curto espaço de tempo; aplicada e avaliada com alunos de pós-graduação lato sensu).

\section{Metodologia}

Esse trabalho relata o desenvolvimento de uma atividade pedagógica, classificada como dinâmica vivencial, que visa ensinar os conceitos de GP executando um projeto de software completo. A atividade é utilizada como ferramenta complementar a outras abordagens, a fim de aplicar os conceitos e, dessa forma, ajudar os alunos a reforçarem o conhecimento e entendimento do assunto.

O desenvolvimento da unidade instrucional seguiu o modelo ISD (Instructional Systems Design) de Dick e Carey (2000), composto por 10 etapas: i) identificar objetivos instrucionais; ii) conduzir análise instrucional; iii) identificar perfil dos participantes; iv) escrever objetivos de desempenho; v) desenvolver instrumentos de avaliação; vi) desenvolver estratégia instrucional; vii) desenvolver e selecionar materiais; viii) projetar e conduzir avaliações formativas; ix) avaliar e conduzir avaliações somativas; e x) revisar instrução.

A atividade foi aplicada em duas turmas da disciplina de Gerenciamento de Projetos, com carga horária de 45 horas/aula, do curso de pós-graduação lato sensu em Engenharia de Software da Universidade do Estado de Santa Catarina (UDESC), nos anos de 2014 e 2015. Para avaliação da atividade, foi aplicado um questionário pós-teste para os alunos participantes, adaptado de Cidral (2003), por ser a abordagem mais próxima encontrada em termos de avaliação e Savi (2011), utilizando a avaliação da aprendizagem com base na taxonomia de Bloom.

Foram respondidos 35 questionários, sendo 19 da turma 2014 e 16 da turma 2015. A avaliação foi composta de 40 perguntas, seguindo metodologia proposta por Cidral (2003), utilizando a escala likert com quatro possibilidades de respostas cada uma: i) discordo inteiramente; ii) discordo parcialmente; iii) concordo parcialmente; iv) concordo inteiramente. As perguntas foram divididas nas seguintes categorias: i) propósito geral; ii) natureza dos problemas; iii) relação dos papéis com problemas; iv) controle dos eventos; v) formato; vi) sequência dos eventos; vii) materiais; viii) feedback e; ix) coordenador. 
Para avaliação da aprendizagem foi utilizada uma auto avaliação de diversos conceitos de GP, na qual os alunos indicaram um valor de 1 a 5 para o conhecimento, entendimento e aplicação dos conceitos antes e depois da realização da atividade.

$\mathrm{Na}$ próxima seção serão descritos os detalhes da unidade instrucional e da dinâmica vivencial Mão na Massa proposta.

\section{A Dinâmica Vivencial Mão na Massa}

A dinâmica Mão na Massa é uma atividade vivencial que visa aplicar os conceitos de gerenciamento de projetos, baseado no PMBOK (PMI, 2014), em um projeto de software que os próprios alunos desenvolvem.

A atividade tem como público alvo estudantes de cursos de graduação e pósgraduação. Como objetivos de aprendizagem principais, tem-se: i) executar as atividades e utilizar os artefatos das atividades de gerenciamento de projetos; ii) vivenciar problemas reais e aplicar conceitos de GP para solucioná-los; iii) utilizar ferramentas de gestão de projetos; iv) entender a dinâmica de trabalho em equipe e a importância da gestão; v) entender o processo de interação com cliente e importância da comunicação.

Para o planejamento da unidade instrucional, foram realizadas as seguintes etapas do modelo de Dick e Carey (2000): i) identificar metas instrucionais: as metas instrucionais foram identificadas, seguindo o objetivo pedagógico do curso e andamento da turma. A meta almejada para a unidade foi que os alunos consigam desenvolver um projeto de software seguindo práticas gerenciamento de projetos baseado no PMBOK; ii) conduzir análise instrucional: identificou-se que, para atingir as metas, os alunos precisariam entender os conceitos básicos de gerenciamento de projetos e saber aplicálos utilizando práticas e artefatos baseados no guia PMBOK; iii) escrever objetivos de desempenho: utilizando a técnica GQM (Goal - Question - Metric) (Basili, Caldiera e Rombach, 1994), foram identificados as seguintes objetivos a serem alcançados pelos alunos: aplicar técnicas de gerenciamento de projetos para gerenciar um projeto de software, como gerente de projetos; desenvolver artefatos para apoiar o gerenciamento de projetos de software e entender a participação com membro da equipe em um projeto de software; iv) analisar aprendizes e contextos: foi realizada por meio de uma enquete com a turma, para identificar o contexto atual da turma, identificando a experiência e conhecimento prévio dos alunos, tanto em gerenciamento de projetos quanto em desenvolvimento de software. Foi identificado que todos os alunos tinham experiência em desenvolvimento de software, a maioria no papel de programador, mas a minoria tinha conhecimento ou experiência no uso de práticas e técnicas de GP.

No projeto e desenvolvimento da dinâmica foram desenvolvidos os planos de aula, instrumentos de avaliação e os materiais didáticos utilizados. Seguindo o modelo de Dick e Carey (2000), foram realizadas as seguintes etapas: i) desenvolvimento da estratégia instrucional; ii) desenvolvimento dos instrumentos de avaliação; e iii) desenvolvimento e seleção de materiais instrucionais.

\subsubsection{Desenvolvimento da Estratégia Instrucional}

A unidade instrucional foi planejada contemplando a explicação introdutória aos conceitos de GP, e a aplicação da dinâmica em paralelo com aulas expositivas, 
demonstrando a teoria a ser aplicada em cada fase da atividade. O Quadro 1 apresenta o cronograma previsto para a unidade.

Quadro 1: cronograma da unidade instrucional

\section{Atividade}

Carga horária

\begin{tabular}{|l|c|}
\hline \multicolumn{2}{|c|}{ Aulas expositivas } \\
\hline Apresentação, motivação e introdução a gerenciamento de projetos & 3 horas \\
\hline Iniciação & 1 hora \\
\hline Planejamento & 4 horas \\
\hline Execução, Monitoramento e Controle & 2 horas \\
\hline Encerramento e Apresentação & 1 hora \\
\hline \multicolumn{2}{|c|}{ Dinâmica Mão na Massa } \\
\hline Desenvolvimento do projeto $\quad$ Fechamento da unidade \\
\hline $\begin{array}{l}\mid \\
\text { Avaliação e feedbadk }\end{array}$ & 5 horas \\
\hline
\end{tabular}

Conforme mostrado no Quadro 1, percebe-se que a disciplina teve um total de 16 horas de teoria e 29 de prática, enfatizando a importância que foi dada para a atividade aplicada. Durante as atividades práticas, o professor deve estar disponível e interagir com os alunos, a fim de esclarecer dúvidas, apoiar e gerenciar o andamento da atividade.

\subsubsection{Desenvolvimento e Seleção de Materiais Instrucionais}

Para a aula expositiva, foram desenvolvidos slides com o conteúdo previsto no plano de aula. Esses slides foram baseados nos grupos de processos, áreas, atividades e práticas do guia PMBOK.

Para a dinâmica vivencial Mão na Massa foram desenvolvidos artefatos para apoiar o gerenciamento do projeto. Esses artefatos foram desenvolvidos em documentos textos e planilhas, a fim de não restringir ao uso de alguma ferramenta específica. Os artefatos desenvolvidos foram: i) termo de abertura; ii) cronograma; iii) plano de gerenciamento de projetos; iv) solicitação de mudança; v) status report; vi) lições aprendidas; vii) termo de encerramento/entregas. $\mathrm{O}$ documento do plano de gerenciamento de projetos contempla todos os planos das demais áreas do PMBOK: escopo, tempo, custo, qualidade, recursos humanos, riscos, integração, partes interessadas, aquisição e comunicação.

\subsubsection{Desenvolvimento dos Instrumentos de Avaliação}

Para cada objetivo de aprendizagem, foram definidas metas e questões a serem avaliadas, utilizando a técnica GQM:

a) executar as atividades e utilizar os artefatos das atividades de gerenciamento de projetos: - Os produtos de gerenciamento foram desenvolvidos e de forma correta? - $\mathrm{O}$ resultado final do produto foi satisfatório?

b) vivenciar problemas reais e aplicar conceitos de GP para solucioná-los: - Os estudantes souberam contornar situações adversas? - Como foi a vivência como gestor de projetos?

c) utilizar ferramentas de gestão de projetos; - Os estudantes souberam elaborar os artefatos de GP de maneira correta?

d) entender a dinâmica de trabalho em equipe e a importância da gestão; - As equipes gerenciaram suas atividades de forma eficaz? 
e) entender o processo de interação com cliente e importância da comunicação: $\mathrm{O}$ aluno soube negociar e interagir com sua equipe e cliente?

Como resultado, chegou-se a um instrumento com critérios e pesos para avaliação da dinâmica (métricas), conforme Tabela 1.

\section{Tabela 1: Instrumento de Avaliação da Dinâmica}

\begin{tabular}{l|c}
\hline Critério & Peso \\
\hline Produto (aspectos relacionados ao software gerado e artefatos de GP) & 20 \\
\hline Escopo (entrega do escopo negociado e atividades de GP desenvolvidas) & 10 \\
\hline Qualidade (qualidade do software e uso corretos das práticas de GP) & 10 \\
\hline Satisfação (referente à comunicação e interação com cliente e equipe) & 20 \\
\hline Cliente (comunicação, negociação) & 10 \\
\hline Equipe (motivação, sinergia, gestão, desempenho) & 10 \\
\hline Execução do Projeto & 60 \\
\hline Organização da equipe (comunicação, distribuição de papeis) & 15 \\
\hline Metodologia e artefatos (informações do projeto usando técnicas corretamente, & 15 \\
completude e qualidade dos artefatos entregues) & 15 \\
\hline Participação (envolvimento individual, nível de entendimento dos conceitos de GP) & 15 \\
\hline Atuação de gerência (comunicação, gestão das pessoas, negociação com cliente) & \\
\hline
\end{tabular}

A avaliação deve ocorrer no final da dinâmica. Para medir a satisfação da equipe foi desenvolvimento um instrumento de auto-avaliação, contemplando os itens: i) motivação; ii) desempenho; iii) sinergia; iv) organização/gerência. Todos os participantes devem responder cada item, seguindo uma escala Likert de 0 a 5, possibilitando gerar a média de cada equipe.

\subsection{A Estrutura da Dinâmica Vivencial Mão na Massa}

Os alunos devem ser divididos em grupos de 5 a 6 pessoas. As equipes podem definir os papeis a serem utilizados, porém o papel de Gerente de Projetos é obrigatório e deverá ser desempenhado por todos os membros da equipe.

A atividade consiste no desenvolvimento de um projeto de software, seguindo um escopo pré-determinado pelo professor, que fará o papel de cliente do projeto. $\mathrm{O}$ escopo dos projetos foi desenvolvido para ser desafiador e diferente para cada equipe, a fim mostrar diversidades de situações em diferentes projetos. O escopo sugerido foi de uma solução que permita aos pais de bebês e crianças registrarem o acompanhamento de seus filhos via Internet. Nesse contexto, o escopo foi dividido em 4 subprojetos, sendo que cada equipe desenvolvesse um deles:

a) Calendário de vacinas: sistema que permite pré-configurar as vacinas necessárias por idade e sexo e manter um registro da vacinação do bebê, enviando alertas antes das datas de vacinação;

b) Acompanhamento de peso e altura: sistema que permite o registro de peso e altura em datas específicas e comparar com as curvas de crescimento padrão, de acordo com o sexo, mostrando gráficos comparativos;

c) Histórico de saúde: sistema que permita o registro de doenças, medicações, dentições, consultas médicas, alergias e outras ocorrências de saúde da criança;

d) Momentos marcantes: sistema que permita registrar os momentos marcantes e fotos do desenvolvimento da criança, como: primeiros passos, primeiras palavras, primeiro banho de mar, além de datas comemorativas. 
A fim de enfatizar as práticas de GP, não foram definidos aspectos técnicos, como: linguagem de programação, banco de dados, etc. A execução do projeto fica sob inteira responsabilidade da equipe. A equipe poderá optar pelo ciclo de vida mais apropriado, pela tecnologia e pela metodologia a ser seguida. Porém, sugere-se que sejam utilizadas as técnicas e artefatos de GP, com obrigatoriedade para: i) termo de abertura; ii) documento de aprovação do escopo; iii) planejamento de custo, tempo e recursos humanos, riscos; iv) monitoramento através de métricas (SPI - Índice de Desempenho de Prazo, CPI - Índice de Desempenho de Custo); v) documento de aceitação de entrega e vi) termo de encerramento.

O professor desempenha o papel de cliente, estando disponível para tirar dúvidas do escopo, fazer negociações, etc. A cada período de 4 horas troca-se o Gerente de Projetos, havendo um rodízio que deve contemplar todos os participantes das equipes. Em cada período deverá ser entregue um relatório do andamento, a ser apresentado pelo gerente de projetos.

Ao final, as equipes devem apresentar os projetos, contemplando aspectos de gerenciamento de projetos (indicadores, evolução, lições aprendidas, etc.) e também o produto gerado (funcionalidades, interface, funcionamento, etc.).

A última etapa da dinâmica é a avaliação, que é realizada pelo professor, baseada nos critérios definidos e também a discussão/feedback da atividade, onde deve-se promover uma discussão com os alunos, a fim de identificar situações de aprendizagem, problemas ocorridos, importância da comunicação e das atividades de gerenciamento, resultados obtidos, entre outros aspectos que se julgar necessário.

\section{Resultados}

Nas duas turmas aplicadas, a atividade foi realizada dividindo as turmas em quatro equipes de cinco alunos. Como resultado, além do ambiente de integração e comprometimento, todas as equipes entregaram os projetos ao final, com softwares prontos para utilização e perfazendo a totalidade ou grande parte dos requisitos iniciais.

Considerando as respostas dos 35 questionários, percebeu-se que quase $75 \%$ dos participantes concordaram fortemente com as afirmações da avaliação, sendo que apenas cerca de $5 \%$ das respostas foram negativas, conforme Tabela 2 , que exibe o resultado por cada uma das nove categorias avaliadas.

A categoria Desenvolvimento Pessoal, refere-se à percepção do aluno com relação ao desenvolvimento de habilidades pessoais para análise e solução de problemas. A categoria Natureza dos Problemas avalia a percepção dos alunos sobre os desafios e problemas abordados na atividade. A categoria Relação dos Papeis com Problemas avalia a percepção dos alunos sobre os papeis que desempenharam no desenvolvimento do projeto de software. Já a categoria Controle dos Eventos avalia a percepção dos alunos sobre a autonomia de tomada de decisão durante a atividade e a consequência de suas escolhas. A categoria Formato avalia a percepção dos alunos sobre a estrutura da atividade e motivação. A categoria Sequência dos Eventos avalia se o fluxo da atividade foi similar a uma situação real. A categoria Materiais avalia a percepção dos alunos sobre as informações e materiais da atividade. A categoria Feedback avalia a percepção dos alunos sobre a reflexão e discussão dos resultados da atividade e, por fim, a 
categoria Coordenador avalia a percepção dos alunos sobre o papel desempenhado pelo professor na atividade, no papel de coordenador da dinâmica.

Tabela 2: Resultados da avaliação por categoria

\begin{tabular}{l|cccc}
\hline Categoria & $\begin{array}{c}\text { Discordo } \\
\text { Inteiramente }\end{array}$ & $\begin{array}{c}\text { Discordo } \\
\text { Parcialmente }\end{array}$ & $\begin{array}{c}\text { Concordo } \\
\text { Parcialmente }\end{array}$ & $\begin{array}{c}\text { Concordo } \\
\text { Inteiramente }\end{array}$ \\
\hline $\begin{array}{l}\text { Desenvolvimento Pessoal } \\
\text { Natureza dos Problemas }\end{array}$ & $0,0 \%$ & $0,0 \%$ & $12,0 \%$ & $88,0 \%$ \\
Relação dos papeis & $1,3 \%$ & $1,4 \%$ & $24,4 \%$ & $64,6 \%$ \\
problemas & $0,0 \%$ & $0,0 \%$ & $20,0 \%$ & $80,0 \%$ \\
Controle dos Eventos & & & & \\
Formato & $0,0 \%$ & $0,2 \%$ & $17,9 \%$ & $81,4 \%$ \\
Sequência dos Eventos & $0,0 \%$ & $0,3 \%$ & $20,0 \%$ & $78,9 \%$ \\
Materiais & $0,0 \%$ & $0,0 \%$ & $20,0 \%$ & $80,0 \%$ \\
Feedback & $0,0 \%$ & $0,0 \%$ & $28,6 \%$ & $71,4 \%$ \\
Coordenador & $0,0 \%$ & $1,1 \%$ & $30,0 \%$ & $65,7 \%$ \\
TOTAL & $1,7 \%$ & $1,9 \%$ & $26,4 \%$ & $58,9 \%$ \\
\end{tabular}

Com relação à aprendizagem, foi solicitado aos alunos uma auto avaliação com relação ao seu avanço no conhecimento pré e pós atividade (de 1 a 5), considerando três dos níveis de aprendizagem da Taxonomia de Bloom (conhecer, entender e aplicar). Os resultados são mostrados na Tabela 3, mostrando um significativo nível de concordância com a contribuição da atividade para a evolução da aprendizagem.

Tabela 3: Evolução da Aprendizagem

\begin{tabular}{|c|c|c|c|c|c|c|c|c|c|}
\hline \multirow[t]{2}{*}{ Conceitos } & \multicolumn{3}{|c|}{ Lembrar o que é } & \multicolumn{3}{|c|}{$\begin{array}{c}\text { Compreender como } \\
\text { funciona }\end{array}$} & \multicolumn{3}{|c|}{ Aplicar na prática } \\
\hline & Antes & Depois & Dif. & Antes & Depois & Dif. & Antes & Depoi & Dif. \\
\hline Iniciação de projeto & 2,06 & 4,26 & 2,20 & 2,00 & 4,20 & 2,20 & 1,60 & 3,89 & 2,29 \\
\hline Planejamento de Escopo & 2,37 & 4,40 & 2,03 & 2,14 & 4,17 & 2,03 & 1,86 & 4,03 & 2,17 \\
\hline Planejamento de Tempo & 2,51 & 4,26 & 1,74 & 2,20 & 4,26 & 2,06 & 1,97 & 4,11 & 2,14 \\
\hline Planejamento de Custo & 2,06 & 4,03 & 1,97 & 1,91 & 3,77 & 1,86 & 1,49 & 3,63 & 2,14 \\
\hline Planejamento de Qualidade & 1,94 & 3,94 & 2,00 & 1,91 & 3,86 & 1,94 & 1,86 & 3,69 & 1,83 \\
\hline Planejamento de Riscos & 2,03 & 4,11 & 2,09 & 1,89 & 4,23 & 2,34 & 1,51 & 3,89 & 2,37 \\
\hline Planejamento de RH & 1,86 & 3,69 & 1,83 & 1,71 & 3,60 & 1,89 & 1,54 & 3,40 & 1,86 \\
\hline Planejamento de Partes & 1,97 & 3,83 & 1,86 & 1,77 & 3,77 & 2,00 & 1,60 & 3,66 & 2,06 \\
\hline Plane & 1,77 & 3,71 & 1,94 & 1,71 & 3,69 & 1,97 & 1,54 & 3,54 & 2,00 \\
\hline Plane & 1,66 & 3,51 & 1,86 & 1,60 & 3,49 & 1,89 & 1,37 & 3,29 & 1,91 \\
\hline Planejamento & 1,97 & 3,83 & 1,86 & 1,83 & 3,83 & 2,00 & 1,60 & 3,71 & 2,11 \\
\hline Gerenciamento da execução do & 2,14 & 4,26 & 2,11 & 2,00 & 4,20 & 2,20 & 1,74 & 3,94 & 2,20 \\
\hline Acompanhamento e & 2,14 & 4,29 & 2,14 & 2,00 & 4,29 & 2,29 & 1,83 & 4,06 & 2,23 \\
\hline Encerramento do projeto & 1,91 & 4,11 & 2,20 & 1,77 & 4,17 & 2,40 & 1,63 & 4,03 & 2,40 \\
\hline
\end{tabular}

Calculando-se a média total de cada nível de aprendizagem, percebe-se uma evolução média significativa tanto no conhecimento $(1,99)$, entendimento $(2,08)$ e aplicação $(2,12)$. Os resultados das avaliações sugerem que a atividade teve uma boa aceitação pelos alunos, sendo que quase a totalidade das respostas foi positiva $(95,3 \%$ de concordância total ou parcial com os quesitos avaliados). Além disso, segundo a auto avaliação dos alunos, a dinâmica vivencial Mão na Massa contribuiu significativamente para a aprendizagem, nos três níveis de aprendizagem (conhecimento, entendimento e aplicação) e em todos os conceitos avaliados, destacando-se: iniciação do projeto (evolução $=2,29)$, planejamento de riscos (evolução $=2,37$ ), gerenciamento da 
execução do projeto (evolução $=2,20$ ), acompanhamento e monitoramento (evolução $=$ 2,29 ) e encerramento do projeto (evolução $=2,40$ ).

Além desses resultados, foi solicitado aos alunos que descrevessem pontos fortes, pontos fracos e sugestões de melhoria da atividade. Dos pontos positivos, destacaramse: i) a vinculação da prática com teoria; ii) utilização de um projeto completo similar a um contexto real e iii) trabalho em equipe. Os pontos negativos mais citados foram: i) pouco tempo para realizar as atividades; ii) defasagem do conteúdo teórico, devido a priorização da atividade na disciplina; iii) cada membro não vivencia todas as situações como gerente de projetos, devido ao rodízio. As sugestões de melhorias mais frequentes foram: i) definição melhor das entregas e artefatos; ii) inserir mais restrições; iii) mais ênfase no controle e acompanhamento do projeto.

\section{Considerações Finais}

O artigo descreveu uma dinâmica vivencial para apoiar no ensino da disciplina de GP. A atividade consistiu no desenvolvimento de quatro projetos de software, sendo uma para cada equipe, no decorrer de uma disciplina em duas turmas de especialização. Todos os 8 projetos foram entregues ao final da disciplina, de maneira satisfatória, sendo que a mesma teve 45 horas/aula concentradas em apenas uma semana de aula.

Numa avaliação, após a aplicação da atividade, os alunos responderam um questionário que demonstrou resultados positivos com relação à percepção dos participantes em relação à atividade ( $95 \%$ de respostas positivas). Da mesma forma, a evolução da aprendizagem, na percepção dos alunos, foi significativa. Numa escala de 1 a 5, a evolução média descrita foi de 2,06 (1,86 para 3,92).

Além dos resultados expressos em números e comentários dos alunos, o fato das equipes de alunos conseguirem entregar um projeto completo funcionando ao final da disciplina foi uma motivação extra. Ficou claro a motivação e engajamento das equipes, que trabalharam diversas horas extra sala de aula nos seus projetos. Após a entrega e apresentação, durante a reflexão e feedback (última etapa da atividade), muitos alunos relataram estarem impressionados com o desempenho da equipe, sendo que na indústria, um projeto de complexidade similar, talvez seria finalizado em semanas ou até meses.

Apesar dos resultados positivos e promissores, mostrando que atividades vivenciais podem ser uma alternativa viável para melhorar o processo de ensinoaprendizagem de GP, existem algumas ameaças aos resultados desse trabalho. $\mathrm{O}$ fato das avaliações serem auto avaliações podem ter direcionados os alunos para responder de forma displicente ou imparcial. Outra questão é que o baixo número de alunos atingidos e num único contexto pode ter direcionado os resultados, devendo ser realizadas mais aplicações em diferentes contextos para poder generalizar os resultados.

Como sugestão de trabalhos futuros, seria interessante a aplicação da dinâmica Mão na Massa em turmas de graduação, outros cursos de pós-graduação ou mesmo treinamentos da indústria. Além disso, uma sugestão de evolução da dinâmica seria a criação de novos contextos e escopo, englobando as sugestões de melhoria dos alunos.

\section{Referências}

Basili, V., Caldiera G. e Rombach, D. (1994). The Goal Question Metric Paradigm. 
Cano, J. L. Lidon, I. Rebollar, R. Roman, P. Saenz, M. J. (2006) "Student Groups Solving Real-life Projects: a case study of experiential learning”. In Int. J. Engng Ed. v. 22, n. 6, p. 1252-1260. Tempus Publications. 2006.

Cidral, A. (2003) "Metodologia de Aprendizagem Vivencial para o Desenvolvimento de Competências para o Gerenciamento de Projetos na Implementação de Sistemas de Informação". Tese de Doutorado do Programa de Pós-Graduação em Engenharia da Produção da Universidade Federal de Santa Catarina, 2003.

Cook, L. Olson, J. (2006) "The Sky's the Limit: An Activity for Teaching Project Management". In Journal of Management Education, v. 30 n. 3, Jun 2006, p. 404420.

Corrêa, F.R. Barçante, L.C. Alves, C.S.C. Maciel, M.S.D. Souza, F. S. (2011) "A Abordagem Vivencial como Ferramenta Aplicada ao Ensino de Gerenciamento de Projetos". In: XXXIX Congresso Brasileiro de Educação em Engenharia (COBENGE2011). Blumenau/SC.

Ibrahim, I. (2011) "Teaching Project Management for IT Students: Methods and Approach”. In IPEDR vol.13, IACSIT Press, Singapore.

Kolb, D. (1984) "Experiential learning: experience as the source of learning and development". Prentice-Hall, New Jersey.

Dantas, A. Barros, M. e Werner, C. (2004) "A Simulation-Based Game for Project Management Experiential Learning”. In Proceedings of the Sixteenth International Conference on Software Engineering and Knowledge Engineering (SEKE'04).

NTL Institute for Applied Behavioral Science, Learning Pyramid. 300 N. Lee Street, Suite 300, Alexandria, VA 22314. 1-800-777-5227. Disponível em: $<$ http://homepages.gold.ac.uk/polovina/learnpyramid/about.htm>.

Ojiako, U. Ashleigh, M. Chipulu, M. Maguire, S. (2011) "Learning and teaching challenges in project management". In International Journal of Project Management, v. 29, n. 3, Abril 2011, p. 268-278.

Petri, G., Marcon Júnior, R.P. (2014). "Um Jogo Educacional Para o Ensino de Metodologias Ágeis". FEES - Fórum de Educação em Engenharia de Software, evento integrante do XXIII Simpósio Brasileiro de Engenharia de Software (SBES),

PMI. Project Management Institute (2014). Um Guia do Conhecimento em

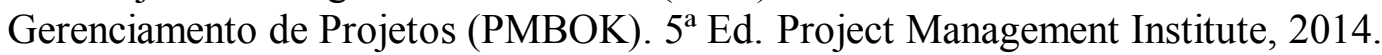

Savi, R. (2011) "Avaliação de Jogos Voltados para a Disseminação do Conhecimento". Tese (doutorado) - Universidade Federal de Santa Catarina. Programa de PósGraduação em Engenharia e Gestão do Conhecimento. Florianópolis, SC, 2011.

Schoeffel, P. (2014). PizzaMia: Dinâmica Vivencial para Apoio ao Ensino de Gerenciamento de Projetos Baseado no PMBOK. In XXII Workshop de Educação em Computação (WEI 2014).

The Standish Group (2013) "Chaos Manifesto 2013". The Standish Group International. Disponível em: < http://versionone.com/ assets/img/files/ CHAOSManifesto2013.pdf>. Acessado em: 20 jan 2016. 